\title{
NEAR OPTIMAL GEOMETRIC IMAGE SCALING USING OBLIQUE PROJECTION OPERATORS
}

\author{
Chulhee Lee, Murray Eden, and Michael Unser \\ National Institutes of Health, BEIP/NCRR,Bldg 13, Room 3W54, \\ Bethesda, Maryland 20892, USA. Email:chulhee@ helix.nih.gov
}

\begin{abstract}
In this paper, we propose to re-size images using an oblique projection operator instead of the orthogonal one in order to obtain faster, simpler, and more general algorithms. The main advantage is that it becomes perfectly feasible to use higher order models (e.g, splines of degree $n \geq 3$ ). We develop the theoretical background and present a simple and practical implementation procedure that uses B-splines. Experiments show that the proposed algorithm consistently outperforms the standard interpolation method and that it essentially provides the same performance as the optimal procedure (least squares solution) with considerably less computations.
\end{abstract}

\section{INTRODUCTION}

Image scaling (magnification and reduction) is an important operation in digital image processing. For example, resolution conversion is required on a routine basis for medical imaging, multimedia, and digital photography. The standard interpolation approach is to fit the digital image with a continuous model and resample this function on a new sampling grid [1]. Nearest neighbor and bilinear interpolation are simplest and fastest, but they produces images which are either blocky or over-smoothed. Better results can be obtained by using higher order models. However, these interpolation methods are sub-optimal since they are not designed to minimize information loss. Recently, Unser et al. proposed a minimum loss image scaling algorithm which applies a continuously-defined prefilter prior to sampling [2]. This algorithm performs the orthogonal projection of the scaled image into a given spline subspace and provides the minimum error approximation. However, it is difficult to obtain a practical numerical implementation as the order of splines increases $(n>1)$.

In this paper, we extend this approach by using oblique projection operators instead of orthogonal ones. Our primary motivation is to design faster and simpler algorithms without sacrificing performance. In particular, we show that we can obtain almost the same performance as in the orthogonal case if we use the same approximation space as before but a much simpler prefilter (box function). In addition, we can also obtain exact implementation formulas for higher order spline models $(n>1)$. Finally, we note that the present theoretical formulation is general enough to include both interpolation and least squares methods as particular cases.

\section{PROJECTION-BASED IMAGE RESIZING}

\section{A. Basic Principle of the Algorithm}

Generally, we can re-size an image (or volume) by successive 1-D processing along the several dimensions of the data. Thus, the mathematical problem reduces to that of re-sizing a one-dimensional signal $s(k), k=0, \ldots N-1$. For our purposes, it is advantageous to think of this process in terms of the following paradigm:

(i) The discrete one-dimensional data set $s(k)$ is fitted with a continuously-defined function $g(x)$ that provides an exact interpolation; that is, such that $\left.g(x)\right|_{x=k}=s(k)$.

(ii) The scaling transformation, which is a mapping from $L_{2}$ into itself, is applied to the function $g(x)$. This yields the continuously-defined rescaled function $f(x)=g(x / a)$ where $a$ is the scaling factor.

(iii) The function $f(x)$ is resampled at the integers (standard approach), or alternatively, is represented by an appropriate approximation of it in a given sampling space (new approach).

The re-sizing method that we propose here only differs from the standard interpolation approach by the way in which we implement step (iii). Instead of a straightforward re-sampling, we will approximate $f(x)$ by its projection $\tilde{f}(x)$ into a given Hilbert space $V(\varphi)$. The re-sized digital signal will then correspond to the samples of $\tilde{f}(x)$; this projected 
function may be thought off as the "aliasing-free" version of $f(x)$.

\section{B. Continuous/Discrete Signal Representation}

We choose to represent our signals in the space $V(\varphi) \subset L_{2}$ which is defined as

$V(\varphi)=\left\{s(x)=\sum_{k \in Z} c(k) \varphi(x-k) \mid c \in l_{2}\right\}$

where $l_{2}$ is the vector space of square-summable sequences. The only restriction on the choice of the generating function $\varphi(x)$ is that the set $\{\varphi(x-k)\}_{k \in Z}$ is a Riesz basis of $V(\varphi)$; this is equivalent to the condition

$$
0<A \leq \widehat{a}_{\varphi}(\omega)=\sum_{k \in Z}|\widehat{\varphi}(\omega+2 \pi k)|^{2} \leq B<+\infty \text { a.e. }
$$

where $\hat{\varphi}(\omega)$ is the Fourier transform of $\varphi(x)$, and where the constants $A$ and $B$ are the so-called frame (or Riesz) bounds [3]. This constraint ensures that the integer shifts of $\varphi$ are linearly independent and that each function $s(x)$ in $V(\varphi)$ is uniquely characterized by the sequence of its coefficients $c(k)$. Note that this type of representation includes splines and wavelets as particular cases.

\section{Oblique Projection}

In order to approximate the continuously defined function $f(x)=g(x / a)$ in $V(\varphi)$, we propose to use an oblique projection. Fig. 1 illustrates the generalized sampling procedure initially proposed in [4]. In this approach, there is no restriction on the prefilter $\varphi_{1}$, which can be chosen independent of the generating function $\varphi_{2}=\varphi$. To compensate for a possible mismatch, the system includes a digital correction filter that ensures that the input signal $f(x)$ and its approximation $\tilde{f}(x)$ are consistent in the sense that they yield the same measurements:

$$
c_{1}(k):=<f(x), \varphi_{1}(x-k)>=<\tilde{f}(x), \varphi_{1}(x-k)>.
$$

Under these conditions, the approximation $\tilde{f}(x)$ corresponds to the projection of $f(x)$ onto $V\left(\varphi_{2}\right)$ perpendicular to the analysis space $V\left(\varphi_{1}\right)$. It is given by

$$
\tilde{f}(x)=P_{2 \perp 1} f(x)=\sum_{k \in Z}\left(q * c_{1}\right)(k) \varphi_{2}(x-k)
$$

where $q=\left(a_{12}\right)^{-1}$ is the convolution inverse of the crosscorrelation sequence $a_{12}(k):=<\varphi_{1}(x-k), \varphi_{2}(x)>$. Note that unless $\varphi_{1} \in V\left(\varphi_{2}\right)$ (or equivalently $V\left(\varphi_{1}\right)=V\left(\varphi_{2}\right)$ ), the corresponding projection error is not orthogonal to the approximation space $V\left(\varphi_{2}\right)$; hence the term "oblique". This type of approximation is essentially equivalent to the orthogonal one in the sense that we have the following error bound (cf. [4])

$\forall s \in L_{2},\left\|f-P_{2} f\right\| \leq\left\|f-P_{2 \perp 1} f\right\| \leq \frac{1}{\cos \theta_{12}}\left\|f-P_{2} f\right\|$,

where $P_{2} f$ denotes the orthogonal projection of $f$ onto $V\left(\varphi_{2}\right)$ and $\theta_{12}$ is the angle between the subspace $V\left(\varphi_{1}\right)$ and $V\left(\varphi_{2}\right)$. We can also show that the oblique and orthogonal errors are asymptotically as the scaling factor goes to infinity the same provided that $\sum \varphi_{1}(x-k)=1$ [5].

\section{IMPLEMENTATION USING SPLINES}

\section{A. Derivation of the Algorithm}

In our implementation, we select the synthesis function $\varphi_{2}=\varphi_{\text {int }}^{n}$ to be the cardinal spline of any order $n$ [6]. As for the analysis function, we try to choose the simplest $\varphi_{1}$ with the narrowest support such that $\sum \varphi_{1}(x-k)=1$. The first choice that comes to mind is the B-spline of zero order: $\varphi_{1}=\beta^{0}$. A clear advantage of using $\beta^{0}$ is that the required inner products can be computed as simple integrals:

$$
\begin{aligned}
c_{1}(k) & =<g(x / a), \beta^{0}(x-k)> \\
& =\int_{-\infty}^{\infty} g(x / a) \beta^{0}(x-k) d x=\int_{k-1 / 2}^{k+1 / 2} g(x / a) d x .
\end{aligned}
$$

If we assume that the signal interpolant $g(x)$ obtained in step (i) is in the spline space, i.e. $g(x)=\sum_{k} c(k) \beta^{n}(x-k)$, then the integral of $g(x)$ can be expressed as

$$
G(x)=\int_{-\infty}^{x} g(x) d x=\sum_{k} c(k) \int_{-\infty}^{x} \beta^{n}(x-k) d x .
$$

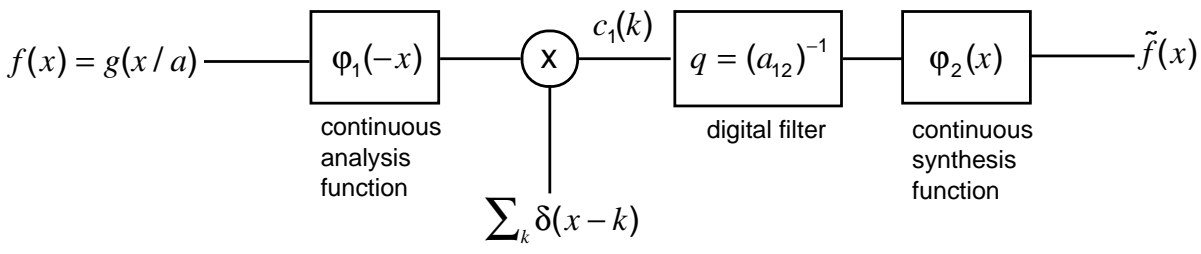

Fig. 1. Generalized sampling using an oblique projection. 
In order calculate this expression explicitly, we use the following proposition.

Proposition 1: The integral of a B-spline of degree $n$ is given by

$$
\int_{-\infty}^{x} \beta^{n}(x) d x=\sum_{k=0}^{\infty} \beta^{n+1}\left(x-\frac{1}{2}-k\right) .
$$

By substituting (5) in (4), we can show that the integral $G(x)$ is a spline of degree $n+1$ :

$$
G(x)=\sum_{k \in Z} s(k) \beta^{n+1}\left(x-\frac{1}{2}-k\right)
$$

whose B-spline coefficients are given by

$$
s(k)=\sum_{l=-\infty}^{k} c(l) .
$$

Equation (3) can therefore be calculated as

$$
\begin{aligned}
c_{1}(k) & =\left\langle g(x / a), \beta^{0}(x-k)\right\rangle \\
& =a G\left(\frac{k}{a}+\frac{1}{2 a}\right)-a G\left(\frac{k}{a}-\frac{1}{2 a}\right),
\end{aligned}
$$

an expression that requires the evaluation of the integral (6) at two particular points. However, we can effectively reduce the computing by half by rewriting $c_{1}(k+1)$ as follows:

$$
c_{1}(k+1)=a G\left(\frac{k+1}{a}+\frac{1}{2 a}\right)-a G\left(\frac{k}{a}+\frac{1}{2 a}\right)
$$

and taking advantage of previous calculations. Finally, for this particular choice of $\varphi_{1}$ and $\varphi_{2}$, we can show that the required digital correction filter is

$$
q(k)=\left(\left(b^{n+1}\right)^{-1} * b^{n}\right)(k)
$$

\section{B. Practical Implementation}

Now we propose the following procedure for image scaling using oblique projection.

\section{Image Scaling Using Oblique Projection}

STEP 1. Find the B-spline representation of the input signal, $g(x)=\sum_{k} c(k) \beta^{n}(x-k)$. This is done efficiently by digital filtering (cf. [7]).

STEP 2. Compute the B-spline coefficients of the integral of $g(x)$ in STEP 1 (cf. (7)).

STEP 3. Calculate the coefficients of the re-scaled function $f(x)=g(x / a)$ (cf. (8)).

STEP 4. Apply the appropriate post-filter to compute $\tilde{f}(k)$ (cf. (1) and (10)).
STEP 1, 2 and 4 are pure digital filtering operations which can be implemented recursively. Computationally, the most expensive part of the algorithm is STEP 3. It is equivalent to a spline interpolation of degree $n+1$. This calculation requires a routine that returns the values of $\beta^{n+1}(x)$ at a given point $x_{0}$; explicit B-spline formulas can be found in [7]. Note that the summation (6) only involves a small number of terms (typically, $n+1$ ) because of the compact support of the basis functions.

\section{RESULTS AND DISCUSSION}

In order to evaluate the proposed algorithm, we performed a succession of complementary image reductions and magnifications, and vice versa. We tested two other algorithms for comparison. The first one is the standard algorithm which fits the image with a spline and then resamples this functions at the appropriate rate. It is one of the most widely used algorithms, at least for lower order splines. For $n=1$, the approach is equivalent to a bilinear interpolation. The second is the optimal method proposed in [2]. It applies the proper pre-filter prior to sampling and provides the minimum error approximation. The global loss of information was measured by the relative mean square difference between the approximation and the initial digital image, expressed in $\mathrm{dB}$.

In a first series of experiments, we first reduced the linear dimension of a brain MR image by $\sqrt{2}$ and then magnified it by $\sqrt{2}$. Table I shows the signal-to-noise ratio as a function of the degree $n$. As expected, the proposed algorithm consistently performed better than the standard interpolation method while requiring comparable processing time. Compared to the least square method, the oblique projection performed comparably with significant decrease in processing time. For the piecewise constant case (splines of degree 0 ), both algorithms are rigorously equivalent. Because an exact closed form formula with the least squares algorithm is extremely difficult for $n>1$, the implemented version of the optimal algorithm for $n=3$ is not numerically exact; it uses a Gaussian approximation to a kernel that is in fact the convolution of two B-splines of different size (cf. [2]). Finally, we note that the first two methods also fit into the general theoretical framework that is presented in Section II. Specifically, if $\varphi_{1} \in V\left(\varphi_{2}\right)$ then we get the least squares solution (orthogonal projection). Likewise, for 
$\varphi_{1}(x)=\delta(x)$ (Dirac), we obtain the standard interpolation procedure - with the important restriction that the angle $\theta_{12}$ between the input and output spaces is not defined any more (i.e., there is no upper error bound in (2)).

Table I. Magnetic resonance image (first reduced by $\sqrt{2}$ and magnified by $\sqrt{2}$ )

\begin{tabular}{||c|c|c|c|}
\hline order & standard & optimal & oblique \\
\hline \hline 0 & $27.19 \mathrm{~dB}$ & $32.73 \mathrm{~dB}$ & $32.73 \mathrm{~dB}$ \\
\hline 1 & $33.65 \mathrm{~dB}$ & $39.25 \mathrm{~dB}$ & $39.02 \mathrm{~dB}$ \\
\hline 3 & $41.54 \mathrm{~dB}$ & $41.74 \mathrm{~dB}$ & $42.15 \mathrm{~dB}$ \\
\hline 5 & $42.20 \mathrm{~dB}$ & & $42.70 \mathrm{~dB}$ \\
\hline 7 & $42.21 \mathrm{~dB}$ & & $42.82 \mathrm{~dB}$ \\
\hline
\end{tabular}

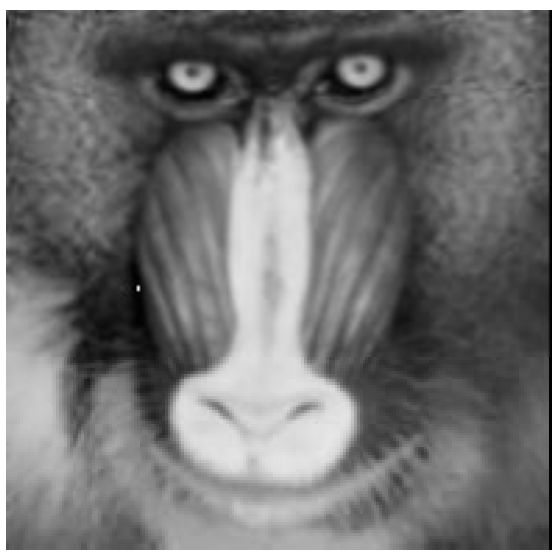

Fig. 2. The standard algorithm $($ degree $=1$, scale $=0.97$, iteration $=10)$

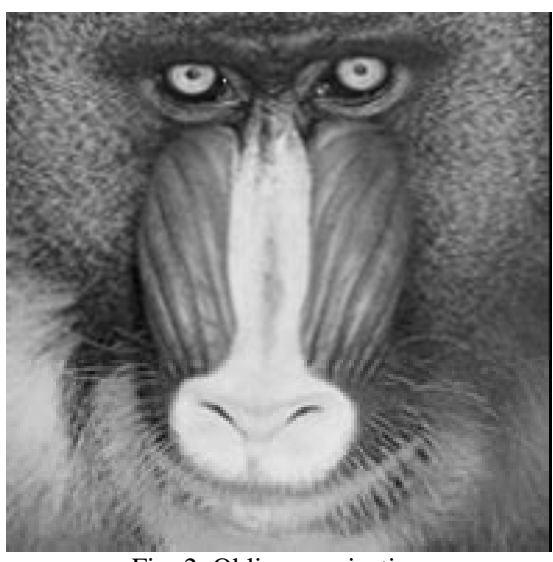

Fig. 2. Oblique projection $($ degree $=1$, scale $=0.97$, iteration $=10)$.

Figs. 2-3 display the final results of 10 cumulative image reduction by a factor of 0.97 using a linear spline signal representation. As can be seen, the oblique projection provides a much higher quality result with a better preservation of small image details.

\section{CONCLUSION}

We propose a new image scaling algorithm based on oblique projection. The oblique projection allows great flexibility in choosing a sampling kernel. In all our test experiments, the proposed algorithm outperformed the conventional interpolation method and showed comparable performance to the optimum algorithm while reducing computing time substantially. The oblique projection that we propose using splines of degree $n$ has approximately the same computational complexity as a spline interpolation of degree $n+1$; but its performance is usually better.

As far as the new algorithm is concerned, the best results for image reduction are obtained for $n=3$ (cubic spline model). For image enlargement, the performance of the algorithm can be improved almost arbitrarily by using higher order splines. Considering that the optimal least squares solution is very difficult to implement for $n>1$, the oblique projection can provide a very attractive solution if high quality results are required.

\section{REFERENCES}

1. Pratt, W.K., Digital image processing. 1978, New York: Wiley.

2. Unser, M., A. Aldroubi, and M. Eden, "Enlargement or reduction of digital images with minimum loss of information," IEEE Trans. Image Processing, Vol. 4, No. 3, pp. 247-258, 1995.

3. Aldroubi, A. and M. Unser, "Sampling procedures in function spaces and asymptotic equivalence with Shannon's sampling theory," Numer. Funct. Anal. and Optimiz., Vol. 15, No. 1\&2, pp. 1-21, 1994.

4. Unser, M. and A. Aldroubi, "A general sampling theory for nonideal acquisition devices," IEEE Trans. Signal Processing, Vol. 42, No. 11, pp. 2915-2925, 1994.

5. Unser, M., "Approximation power of biorthogonal wavelet expansions," IEEE Signal Processing, Vol. No. pp. in press.

6. Aldroubi, A., M. Unser, and M. Eden, "Cardinal spline filters : stability and convergence to the ideal sinc interpolator," Signal Processing, Vol. 28, No. 2, pp. 127-138, 1992.

7. Unser, M., A. Aldroubi, and M. Eden, "B-spline signal processing. Part II : efficient design and applications," IEEE Trans. Signal Processing, Vol. 41, No. 2, pp. 834-848, 1993 